\title{
Effect and Safety of Rosuvastatin in Acute Ischemic Stroke
}

\author{
Ji Hoe Heo, ${ }^{a}$ Dongbeom Song, ${ }^{a}$ Hyo Suk Nam, ${ }^{a}$ Eung Yeop Kim, ${ }^{b}$ Young Dae Kim, ${ }^{a}$ Kyung-Yul Lee, ${ }^{c}$ \\ Ki-Jeong Lee, ${ }^{\mathrm{a}}$ Joonsang Yoo, ${ }^{\mathrm{a}}$ Youn Nam Kim, ${ }^{\mathrm{d}}$ Byung Chul Lee, ${ }^{\mathrm{e}}$ Byung-Woo Yoon, ${ }^{\mathrm{f}}$ Jong S. Kim, ${ }^{\mathrm{g}}$ \\ for the EUREKA Investigators
}

\author{
${ }^{a}$ Department of Neurology, Yonsei University College of Medicine, Seoul, Korea \\ bDepartment of Radiology, Gachon University, Gil Medical Center, Incheon, Korea \\ 'Department of Neurology, Gangnam Severance Hospital, Yonsei University College of Medicine, Seoul, Korea \\ ${ }^{\mathrm{d} C l i n i c a l}$ Trials Center, Severance Hospital, Yonsei University Health System, Seoul, Korea \\ ${ }^{e}$ Department of Neurology, Hallym University Sacred Heart Hospital, Pyeongchon, Korea \\ ${ }^{\mathrm{f}}$ Department of Neurology, Seoul National University College of Medicine, Seoul, Korea \\ ${ }^{g}$ Department of Neurology, University of Ulsan College of Medicine, Asan Medical Center, Seoul, Korea
}

Background and Purpose The benefit of statins in acute stroke remains uncertain. Statins may prevent stroke recurrence during the acute stage of stroke via pleiotropic effects. However, statins may increase the risk of intracerebral hemorrhage. We investigated the effect and safety of rosuvastatin in acute stroke patients.

Methods This randomized, double-blind, multi-center trial compared rosuvastatin $20 \mathrm{mg}$ and placebo in statin-naïve stroke patients who underwent diffusion-weighted imaging (DWI) within 48 hours after symptom onset. The primary outcome was occurrence of new ischemic lesions on DWI at 5 or 14 days.

Results This trial was stopped early after randomization of 316 patients due to slow enrollment. Among 289 patients with at least one follow-up imaging, the frequency of new ischemic lesions on DWI was not different between groups (rosuvastatin: 27/137, 19.7\% vs. placebo: $36 / 152,23.6 \%$ ) (relative risk $0.83,95 \%$ confidence interval $0.53-1.30$ ). Infarct volume growth at 5 days (log-transformed volume change, rosuvastatin: $0.2 \pm 1.0 \mathrm{~mm}^{3}$ vs. placebo: $0.3 \pm 1.3 \mathrm{~mm}^{3} ; P=0.784$ ) was not different, either. However, hemorrhagic infarction or parenchymal/subarachnoid hemorrhage on gradient-recalled echo magnetic resonance imaging occurred less frequently in the rosuvastatin group $(6 / 137,4.4 \%)$ than the placebo group $(22 / 152,14.5 \%, P=0.007)$. Among 314 patients with at least one dose of study medication, progression or clinical recurrence of stroke tended to occur less frequently in the rosuvastatin group $(1 / 155,0.6 \%$ vs. $7 / 159,4.4 \%, P=0.067)$. Adverse events did not differ between groups. Conclusions The efficacy of rosuvastatin in reducing recurrence in acute stroke was inconclusive. However, statin use was safe and reduced hemorrhagic transformation.

\author{
Correspondence: Ji Hoe Heo \\ Department of Neurology, Yonsei \\ University College of Medicine \\ 50-1 Yonsei-ro, Seodaemoon-gu, \\ Seoul 03722, Korea \\ Tel: +82-2-2228-1605 \\ Fax: +82-2-393-0705 \\ E-mail:jhheo@yuhs.ac
}

Received: October 26, 2015 Revised: January 6, 2016 Accepted: January 6, 2016

\begin{abstract}
This trial was supported by a research grant from AstraZeneca and the Korea Healthcare Technology REtD Project, Ministry of Health and Welfare, Republic of Korea (HI08C2149, HI10C2020). The investigators are fully responsible for the design of the study and protocol; conduct of the study; collection, monitoring, management, analyses, and interpretation of data; and writing and submission of the manuscript, without involvement of the sponsors.
\end{abstract}

The authors have no financial conflicts of interest.

Keywords Stroke; Rosuvastatin; Statin; Diffusion-weighted imaging

\section{Introduction}

Statins are effective in primary and secondary prevention of stroke. ${ }^{1-3}$ Their long-term beneficial effects may be primarily mediated by their lipid-lowering effects. Statins may also work effectively in preventing recurrence or progression during the 
acute stage of stroke because they have antithrombotic, antiinflammatory, and anti-oxidative effects. ${ }^{47}$

Several observational studies have suggested that statin use before or during the acute stage of stroke was associated with better functional outcome and reduced mortality. ${ }^{7-9}$ By contrast, intracerebral hemorrhage (ICH) developed more frequently in patients treated with high-dose statins during the subacute or chronic stage of ischemic stroke in large randomized trials. ${ }^{2,10}$ Since the risk of hemorrhagic transformation is greater during the acute stage of stroke, statin treatment in acute stroke raises the concern of increased ICH risk. However, data from randomized trials are insufficient to establish whether statins are effective and safe in patients with acute ischemic stroke. ${ }^{11}$ Furthermore, statins are not recommended in stroke guidelines as an agent with neuroprotective actions to improve outcome in acute stroke. ${ }^{12}$ Therefore, we investigated the effect and safety of rosuvastatin in acute ischemic stroke patients.

\section{Methods}

This double-blind, placebo-controlled, randomized, multicenter study was approved by the Ministry of Food and Drug Safety, Korea, and the institutional review board at each study center. Written informed consent was obtained from each patient. This trial (Effects of very early use of rosuvastatin in preventing recurrence of ischemic stroke [EUREKA]) was registered on ClinicalTrials.gov (NCT01364220).

\section{Study population}

We enrolled patients over 20 years old diagnosed with acute ischemic stroke on diffusion-weighted imaging (DWI) within 48 hours after symptom onset who had been untreated with a statin for the previous 3 months. Patients also should show any degree of stenosis on the relevant artery of infarction on DWI. Patients with hemorrhagic stroke, history of symptomatic hemorrhagic stroke, high-risk cardiac sources of embolism, or stroke of other determined etiology were excluded. Other exclusion criteria are described in Supplementary data.

The modified intention-to-treat population consisted of patients who underwent a baseline magnetic resonance imaging (MRI), had triglyceride (TG) $<500 \mathrm{mg} / \mathrm{dL}$, low-density lipoprotein cholesterol $<190 \mathrm{mg} / \mathrm{dL}$, and took at least one dose of study medication. The per-protocol (PP) population consisted of patients who completed scheduled MRIs without a major protocol violation.

\section{Randomization, blinding, and interventions}

Patients were randomized 1:1 to receive either rosuvastatin or placebo. Permutated-block randomization with a block size of 4 was generated by an independent clinical trials center (Severance Hospital, Yonsei University Health System, Seoul, Korea). After patients were screened and completed enrollment, drugs were assigned a unique study number, selected sequentially from the central randomization list that corresponded to the treatment pack, and allocated in a doubleblind manner. The drug was administered within 18 hours after baseline MRI and then daily during the 14-day treatment period. Patients received either one 20-mg tablet or a placebo tablet, once daily.

\section{Sample size}

We hypothesized that, compared to placebo, rosuvastatin would reduce the occurrence of new ischemic lesions on MRI by $30 \%$. To test our hypothesis, assuming a type I error of $5 \%$ and a power of $80 \%$, sample sizes were calculated as 260 in each group. The proportion in the rosuvastatin group was assumed to be 0.40 under the null hypothesis and 0.28 under the alternative hypothesis, based on a previous study that recognized new ischemic lesions on DWI in 34\%-47.4\% of patients during the first week after baseline DWI taken within 24 hours after symptom onset. ${ }^{13,14}$ The test statistic used was the 2-sided Fisher's exact test. Assuming a drop-out rate of $5 \%$, the total number of patients needed was 547 .

\section{Imaging protocol}

To be eligible, patients underwent DWI, fluid attenuated inversion recovery, gradient-recalled echo (GRE), and magnetic resonance (MR) angiography that included both the circle of Willis and neck vessels at baseline using a $1.5 \mathrm{~T}$ or $3.0 \mathrm{~T} \mathrm{MR}$ scanner. Computed tomography (CT) angiography was also allowed. The follow-up imaging schedule included DWI, GRE, and fluid attenuated inversion recovery at $5 \pm 1$ days and $14 \pm 2$ days using the same MR scanner. The images were saved in Digital Imaging and Communications in Medicine (DICOM) format and sent to the independent clinical trials center for review of adequacy and analyses.

\section{Adjudication of images}

Two stroke neurologists blinded to clinical and group information reviewed angiographic images and determined the presence of relevant artery stenosis in ischemic lesions on DWI. In cases of discrepancy between the reviewers, the decision was made by a third reviewer (a neuroradiologist). The reviewers also measured the degree of stenosis of the relevant 
artery based on methods used in the North American Symptomatic Carotid Endarterectomy Trial for extracranial arteries and the Warfarin and Aspirin for Symptomatic Intracranial Arterial Stenosis trial for intracranial arteries ${ }^{15,16}$ and categorized stenosis as $\geq 50 \%$ or $<50 \%$.

\section{Outcomes}

Imaging outcomes were assessed by 2 reviewers blinded to clinical and group information. The primary outcome was occurrence of a new ischemic lesion on DWI or fluid attenuated inversion recovery at 5 or 14 days. The secondary outcomes were the volume change of ischemic lesions and the percent improvement in National Institute of Health Stroke Scale (NIHSS) at 5 days and 14 days. The safety outcome included adverse events, laboratory results, and the presence of any intracranial hemorrhagic transformation on GRE, which in-

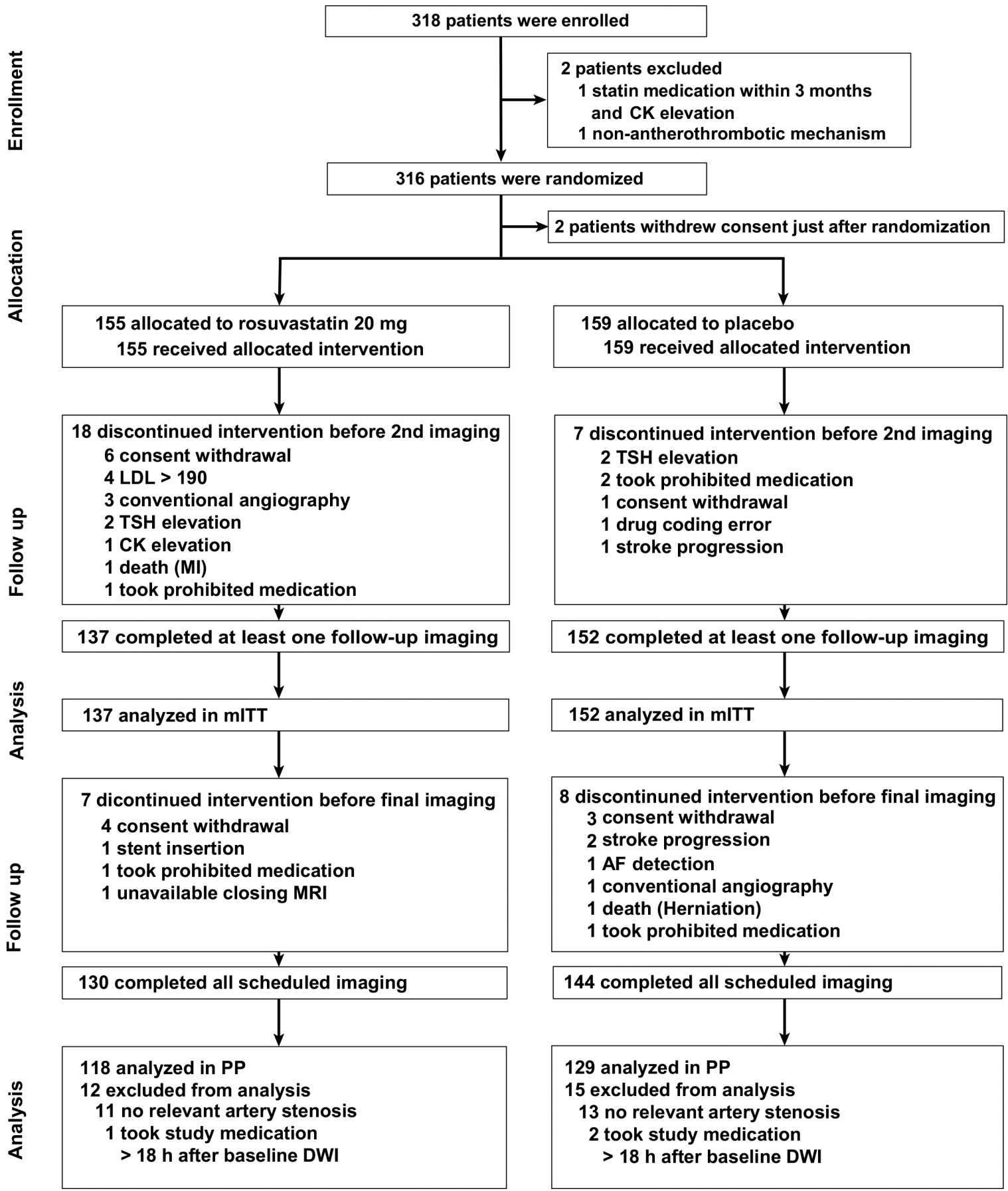

Figure 1. Trial profile. CK, creatine kinase; LDL, low-density lipoprotein; TSH, thyroid-stimulating hormone; mITT, modified intention-to-treat population; $\mathrm{MRI}$, magnetic resonance imaging; PP, per-protocol population; DWI, diffusion-weighted imaging. 
cluded hemorrhagic infarction (HI) and parenchymal hemorrhage based on European Cooperative Acute Stroke Study definitions. ${ }^{17}$ In case of a discrepancy in the presence of new lesions or hemorrhagic transformation, conclusions were reached by consensus. The volume of infarctions was measured on DWI in a semi-automatic manner using Xelis software (Infinitt, Seoul, Korea). The intraclass correlation coefficient between the reviewers for log-transformed volume measurements was 0.99 . The percent improvement was defined as ([NIHSS at 5 days or 14 days - NIHSS at baseline]/NIHSS at baseline) $\times 100 .^{18}$

Details of the conduction of study are provided in Supplemental data.

\section{Statistical analysis}

The efficacy outcome was compared based on the modified intention-to-treat and PP population. Safety was assessed in all patients who took at least one dose of study medication. We used the $\chi^{2}$ test with continuity correction to compare the occurrence of newly developed DWI lesion, the independent sample $t$ test to compare the percent improvement in NIHSS, and the mixed-effect model to compare the change in logtransformed DWI lesion volume between the rosuvastatin and placebo groups. The $\chi^{2}$ test or Fisher's exact test was performed to compare safety outcomes. Statistical analyses were performed using SAS statistical software, version 9.2 (SAS institute Inc., Cary, NC). Data are presented as number (\%) or mean \pm standard deviation. Two-sided $P$ values $<0.05$ were considered statistically significant.

\section{Results}

\section{Baseline characteristics}

A total of 28 centers in Korea participated in this study, and 25 centers enrolled at least 1 patient. Among 318 patients enrolled, 316 patients met inclusion criteria and were randomized, and 314 took at least one dose of study medication (155 in the rosuvastatin group and 159 in the placebo group). Among them, the primary outcome was assessed in 289 patients (Figure 1). Baseline demographic characteristics were similar between the groups except total cholesterol and low-density lipoprotein cholesterol levels, which were higher in the rosuvastatin group (Table 1, Supplemental Table 1). Characteristics of the patients whose primary outcome was not assessed are provided in as a Supplemental Table 2.

\section{Efficacy outcomes}

Efficacy was compared in the modified intention-to-treat
Table 1. Baseline characteristics of the rosuvastatin and placebo groups

\begin{tabular}{|c|c|c|c|}
\hline & $\begin{array}{l}\text { Rosuvastatin } \\
\quad(n=155)\end{array}$ & $\begin{array}{l}\text { Placebo } \\
(\mathrm{n}=159)\end{array}$ & $P$ value \\
\hline \multicolumn{4}{|l|}{ Demographics } \\
\hline Sex (male) & $87(56.1)$ & $101(63.5)$ & 0.222 \\
\hline Age (year) & $65.4 \pm 12.3$ & $64.6 \pm 11.3$ & 0.564 \\
\hline Body mass index $\left(\mathrm{kg} / \mathrm{m}^{2}\right)$ & $23.8 \pm 3.1$ & $24.1 \pm 3.0$ & 0.417 \\
\hline Abdominal circumference $(\mathrm{cm})$ & $86.2 \pm 9.4$ & $87.2 \pm 9.3$ & 0.388 \\
\hline \multicolumn{4}{|l|}{ Past history } \\
\hline Hypertension & $104(67.0)$ & $102(64.1)$ & 0.667 \\
\hline Diabetes mellitus & $50(32.2)$ & $51(32.0)$ & 1.000 \\
\hline Hypercholesterolemia & $24(15.4)$ & $26(16.3)$ & 0.955 \\
\hline Smoking & $72(46.4)$ & $67(42.1)$ & 0.512 \\
\hline Coronary artery occlusive disease & $1(0.6)$ & $4(2.5)$ & 0.371 \\
\hline Peripheral artery occlusive disease & $2(1.2)$ & $0(0.0)$ & 0.243 \\
\hline Previous stroke & $16(10.3)$ & $13(8.1)$ & 0.644 \\
\hline \multicolumn{4}{|l|}{ Concomitant medication } \\
\hline Antihypertensive & $67(43.2)$ & 73 (45.9) & 0.715 \\
\hline Antiplatelet & & & 0.677 \\
\hline Aspirin & $39(25.1)$ & $39(24.5)$ & \\
\hline Clopidogrel & $9(5.8)$ & $10(6.2)$ & \\
\hline Aspirin and clopidogrel & $88(56.7)$ & $92(57.8)$ & \\
\hline Aspirin and cilostazol & $5(3.2)$ & $9(5.6)$ & \\
\hline Aspirin, clopidogrel, and cilostazol & $14(9.0)$ & $9(5.6)$ & \\
\hline Anticoagulant & $0(0.0)$ & $1(0.6)$ & 1.000 \\
\hline Lipid-lowering drug (other than statin) & $2(1.2)$ & $1(0.6)$ & 0.619 \\
\hline Diabetes mellitus drug & $34(21.9)$ & $35(22.0)$ & 1.000 \\
\hline Nonsteroidal anti-inflammatory drug & $8(5.1)$ & $13(8.1)$ & 0.399 \\
\hline $\begin{array}{l}\text { Intravenous tissue plasminogen } \\
\text { activator }\end{array}$ & $4(2.5)$ & $5(3.1)$ & 1.000 \\
\hline $\begin{array}{l}\text { Log-transformed baseline } \\
\text { diffusion-weighted imaging } \\
\text { volume }\left(\mathrm{mm}^{3}\right)\end{array}$ & $6.7 \pm 1.9$ & $6.8 \pm 2.0$ & 0.761 \\
\hline $\begin{array}{l}\text { Baseline National Institute of Health } \\
\text { Stroke Scale }\end{array}$ & $3[1-6]$ & $3[2-5.3]$ & 0.713 \\
\hline Degree of stenosis & & & 0.703 \\
\hline No stenosis & $12(7.7)$ & $12(8.8)$ & \\
\hline$<50 \%$ & $52(36.4)$ & $56(38.6)$ & \\
\hline $50 \%-99 \%$ & 47 (32.9) & $53(36.6)$ & \\
\hline Occlusion & $44(30.8)$ & $36(24.8)$ & \\
\hline \multicolumn{4}{|l|}{ Lab } \\
\hline White blood cells $\left(\times 10^{3} / \mu \mathrm{L}\right)$ & $7.96 \pm 89.2$ & $7.48 \pm 84.3$ & 0.929 \\
\hline Neutrophils $\left(\times 10^{3} / \mu \mathrm{L}\right)$ & $6.24 \pm 1.6$ & $6.13 \pm 1.74$ & 0.616 \\
\hline Hemoglobin $(\mathrm{g} / \mathrm{dL})$ & $14 \pm 1.6$ & $14 \pm 1.6$ & 0.823 \\
\hline Hematocrit (\%) & $41 \pm 4.5$ & $41.1 \pm 4.4$ & 0.817 \\
\hline Platelet count $\left(\times 10^{3} / \mu \mathrm{L}\right)$ & $246.3 \pm 60.3$ & $240.1 \pm 65.5$ & 0.296 \\
\hline Blood urea nitrogen $(\mathrm{mg} / \mathrm{dL})$ & $15.1 \pm 5.2$ & $15.5 \pm 5.6$ & 0.575 \\
\hline Creatinine $(\mathrm{mg} / \mathrm{dL})$ & $0.83 \pm 0.208$ & $0.865 \pm 0.253$ & 0.244 \\
\hline Fasting glucose (mg/dL) & $131.2 \pm 57.8$ & $136.8 \pm 57.1$ & 0.256 \\
\hline Albumin (g/dL) & $4.1 \pm 0.34$ & $4.14 \pm 0.36$ & 0.302 \\
\hline Uric acid (mg/dL) & $5.06 \pm 1.36$ & $5.06 \pm 1.53$ & 0.770 \\
\hline high sensitivity C-reactive protein (mg/dL) & $2.517 \pm 7.111$ & $2.114 \pm 6.794$ & 0.491 \\
\hline Uric acid (mg/dL) & $5.06 \pm 1.36$ & $5.06 \pm 1.53$ & 0.770 \\
\hline high sensitivity C-reactive protein (mg/dL) & $2.517 \pm 7.111$ & $2.114 \pm 6.794$ & 0.491 \\
\hline
\end{tabular}

Values are number $(\%)$, mean \pm standard deviation, or median [interquartile range]. 
population (137 patients in the rosuvastatin group and 152 patients in the placebo group). New ischemic lesions on DWI were observed less frequently in the rosuvastatin group (27 patients $[19.7 \%]$ ) than in the placebo group (36 patients [23.6\%]), but the difference was not statistically significant (absolute difference 3.9\%, relative risk [RR] 0.83, 95\% confidence interval $[\mathrm{CI}]$ 0.53-1.30, $P=0.500$ ) (Figure 2A). Infarction volumes on DWI increased at 5 days and then decreased at 14 days in both groups. Infarct volume growth at 5 days (log-transformed volume change, rosuvastatin: $0.2 \pm 1.0 \mathrm{~mm}^{3}$ vs. placebo: $\left.0.3 \pm 1.3 \mathrm{~mm}^{3} ; P=0.784\right)$ and percent improvement in NIHSS (rosuvastatin vs. placebo: $36.6 \pm 56.7$ vs. 27.1 \pm 90.8 at 5 days, $P=0.282$ and $51.4 \pm 51.6$ vs. $42.7 \pm 91.5$ at 14 days, $P=0.315$ ) were not different.

\section{Safety outcomes}

Of 314 patients, 3 in the placebo group (1.9\%) and none in the rosuvastatin group demonstrated clinical recurrence of ischemic stroke $(P=0.248)$. Progression or clinical recurrence of stroke was reported as a serious adverse event in 7 patients

$\begin{array}{ccc}\text { Entire population } & \text { Rosuvastatin } & \text { Placebo } \\ 5 \text { or 14 days } & 27 / 137(19.7 \%) & 36 / 152(23.6 \%) \\ 5 \text { days } & & \\ 14 \text { days } & & \\ \text { Multiple lesions } & & \\ \text { Yes } & 20 / 78(25.6 \%) & 26 / 80(32.5 \%) \\ \text { No } & 7 / 59(11.9 \%) & 10 / 72(13.9 \%) \\ \text { Relevant artery stenosis ( } \geq 50 \%) & \\ \text { Yes } & 19 / 83(22.9 \%) & 25 / 87(28.7 \%) \\ \text { No } & 8 / 54(14.8 \%) & 11 / 65(16.9 \%)\end{array}$

(4.4\%) in the placebo group, but in only 1 patient $(0.6 \%)$ in the rosuvastatin group $(P=0.067)$. The frequency of adverse events did not differ between the groups (Table 2).

On GRE, HI was observed in 6 patients at baseline (5/155 [3.2\%] in the rosuvastatin and $1 / 159$ [0.65] in the placebo groups). Occurrence of any new intracranial hemorrhagic transformation (HI, parenchymal hemorrhage, or subarachnoid hemorrhage) or aggravation of pre-existing HI1 at baseline (defined as conversion to HI2 or parenchymal hemorrhage) was assessed in 289 patients with available GRE at 5 or 14 days. Any new $\mathrm{HI}$ was observed less frequently in the rosuvastatin group $(6 / 137,4.4 \%)$ than in the placebo group (22/ $152,14.5 \%)(P=0.007)$. In the rosuvastatin group, 1 patient developed parenchymal hemorrhage $(P=0.478)$, and 1 patient developed focal cortical subarachnoid hemorrhage on GRE $(P=0.478)$, both of whom were asymptomatic (Table 3$)$.

\section{Post-hoc subgroup analysis}

We compared the occurrence of a new ischemic lesion on DWI in patients with relevant artery stenosis $\geq 50 \%$. New

\section{Relative risk (95\% Cl)}

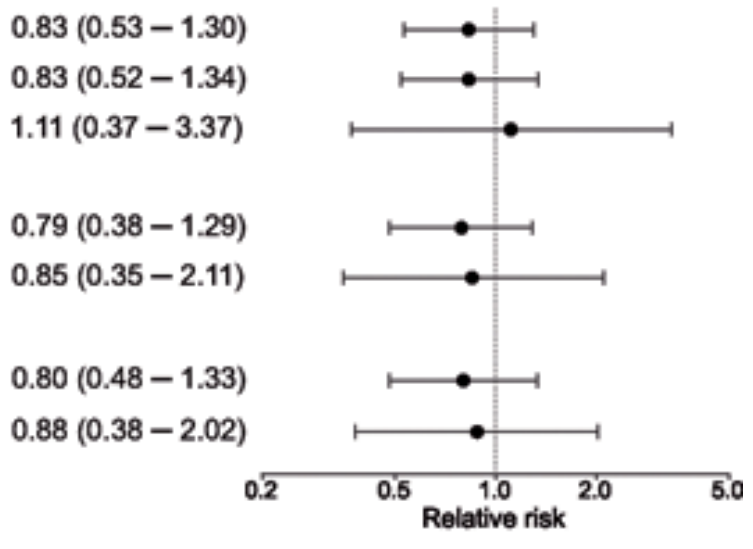

Relative risk (95\% Cl)

Entire population

5 or 14 days

$24 / 118(20.3 \%) \quad 34 / 129(26.3 \%)$

$0.77(0.49-1.22)$

$0.74(0.45-1.21)$

$1.31(0.41-4.19)$

14 days

$0.68(0.41-1.14)$

$0.95(0.38-2.37)$

$5 / 69(36.2 \%)$

$7 / 49(14.3 \%)$

$9 / 60(15.0 \%)$

Relevant artery stenosis ( $\geq 50 \%$ )

$\begin{array}{rrr}\text { Yes } & 17 / 79(26.8 \%) & 24 / 78(30.8 \%) \\ \text { No } & 7 / 59(11.9 \%) & 10 / 51(19.6 \%)\end{array}$

$0.70(0.41-1.20)$

$0.92(0.38-2.10)$ 
Table 2. Adverse events

\begin{tabular}{|c|c|c|c|}
\hline & $\begin{array}{c}\text { Rosuvastatin } \\
(n=155)\end{array}$ & $\begin{array}{l}\text { Placebo } \\
(n=159)\end{array}$ & $P$ value \\
\hline Any $A E$ & $88(56.8)$ & $87(54.7)$ & 0.800 \\
\hline Any SAE & $4(2.6)$ & $8(5.0)$ & 0.379 \\
\hline SAE in nervous system & $2(1.3)$ & $8(5.0)$ & 0.104 \\
\hline $\begin{array}{l}\text { Progression or clinical recurrence of } \\
\text { stroke* }^{*}\end{array}$ & $1(0.6)$ & $7(4.4)$ & 0.067 \\
\hline Brain herniation & $0(0.0)$ & $1(0.6)$ & 1.000 \\
\hline Intracerebral hemorrhage & $1(0.6)$ & $0(0.0)$ & 0.494 \\
\hline SAE in cardiac system & $2(1.2)$ & $1(0.6)$ & 0.619 \\
\hline Atrial fibrillation & $1(0.6)$ & $0(0.0)$ & 0.494 \\
\hline Chest discomfort & $0(0.0)$ & $1(0.6)$ & 1.000 \\
\hline Myocardial infarction & $1(0.6)$ & $0(0.0)$ & 0.494 \\
\hline SAE in gastrointestinal system & $0(0.0)$ & $1(0.6)$ & 1.000 \\
\hline Upper gastrointestinal bleeding & $0(0.0)$ & $1(0.6)$ & 1.000 \\
\hline $\begin{array}{l}\text { Any AE resulting in discontinuation of } \\
\text { study drug }\end{array}$ & $5(2.1)$ & $4(1.9)$ & 0.539 \\
\hline \multicolumn{4}{|l|}{ Any $A E$ with incidence of $\geq 5 \%$} \\
\hline Constipation & $13(8.4)$ & $15(9.4)$ & 0.726 \\
\hline Headache & $9(5.8)$ & $15(9.4)$ & 0.319 \\
\hline $\begin{array}{l}\text { Progression or clinical recurrence of } \\
\text { stroke }^{\dagger}\end{array}$ & $11(7.1)$ & $13(8.2)$ & 0.883 \\
\hline Coronary artery occlusive disease & $16(10.3)$ & $12(7.5)$ & 0.506 \\
\hline Hypertension & $12(7.7)$ & $14(8.8)$ & 0.891 \\
\hline \multicolumn{4}{|l|}{ Musculoskeletal AE } \\
\hline Myalgia & $3(1.9)$ & $4(2.5)$ & 1.000 \\
\hline Myopathy & $0(0.0)$ & $0(0.0)$ & 1.000 \\
\hline Rhabdomyolysis & $0(0.0)$ & $0(0.0)$ & 1.000 \\
\hline \multicolumn{4}{|l|}{ Laboratory values } \\
\hline CK elevation $>3 \times$ ULN & $0(0.0)$ & $0(0.0)$ & 1.000 \\
\hline $\begin{array}{l}\text { Aspartate aminotransferase or alanine } \\
\text { aminotransferase elevation }>3 \times \text { ULN }\end{array}$ & $0(0.0)$ & $0(0.0)$ & 1.000 \\
\hline Death & $1(0.4)$ & $1(0.5)$ & 1.000 \\
\hline
\end{tabular}

Values are number (\%).

*Two patients with clinical recurrent stroke were included; 'Three patients with clinical recurrent stroke (including two patients reported in SAE) were included.

AE, adverse event; SAE, serious adverse event; CK, creatine kinase; ULN, upper limit of the normal range.

ischemic lesions were found in 19 of 83 patients $(22.9 \%)$ in the rosuvastatin group and 25 of 87 patients $(28.7 \%)$ in the placebo group (RR, $0.80 ; 95 \% \mathrm{CI}, 0.48-1.33 ; P=0.387$ ). Twenty of 78 patients $(25.6 \%)$ in the rosuvastatin group and 26 of 80 patients (32.5\%) in the placebo group with multiple lesions on baseline DWI had new lesions (RR, 0.79; 95\% CI, $0.48-1.29 ; P=0.346)$ (Figure 2A).

\section{PP Population}

A total of 118 patients in the rosuvastatin group and $129 \mathrm{pa}-$ tients in the placebo group were included for the PP population (Figure 1). New ischemic lesions were found in 24 patients (20.3\%) in the rosuvastatin group and 34 patients $(26.3 \%)$ in the placebo group (RR 0.77; 95\% CI, 0.49-1.22, $P=0.335$ )
Table 3. Occurrence of intracranial hemorrhagic transformation on gradient-recalled echo (GRE)

\begin{tabular}{lccc}
\hline & $\begin{array}{c}\text { Rosuvastatin } \\
(n=137)\end{array}$ & $\begin{array}{c}\text { Placebo } \\
(n=152)\end{array}$ & Pvalue \\
\hline HI1 & $2(1.4)$ & $15(9.9)$ & 0.002 \\
HI2 & $2^{*}(1.4)$ & $7^{\dagger}(4.6)$ & 0.177 \\
PH1 & $1(0.7)$ & $0(0.0)$ & 0.478 \\
PH2 & $0(0.0)$ & $0(0.0)$ & \\
Radiological subarachnoid hemorrhage & $1(0.7)$ & $0(0.0)$ & 0.478 \\
Any hemorrhagic transformation & $6(4.3)$ & $22(14.5)$ & 0.007
\end{tabular}

Values are number (\%).

Hemorrhagic transformation was categorized into small petechial hemorrhagic infarction (HI1), confluent petechial HI (HI2), small parenchymal hemorrhage (PH1, <30\% of infarct, mild mass effect), and large $\mathrm{PH}(\mathrm{PH} 2,>30 \%$ of infarct, marked mass effect). ${ }^{17}$

* including 1 patient who had HI-1 on baseline GRE and HI-2 on follow-up GRE. 'including 1 patient who had $\mathrm{HI}-1$ on baseline GRE and HI-2 on follow-up GRE, 4 patients who had HI-1 on 5-day GRE and HI-2 on 14-day GRE.

(Figure 2B). In the subgroup with relevant artery stenosis $\geq 50 \%$, new ischemic lesions were detected in 17 of 79 patients $(21.5 \%)$ in the rosuvastatin group and 24 of 78 patients (30.8\%) in the placebo group (RR, 0.70; 95\% CI 0.41-1.20; $P=0.192)$. In the subgroup with multiple lesions, new ischemic lesions were observed in 17 of $69(24.8 \%)$ patients in the rosuvastatin group and 25 of $69(36.2 \%)$ patients in the placebo group (RR 0.68; 95\% CI, 0.41-1.14; $P=0.145$ ) (Figure 2B).

\section{Discussion}

This study was inconclusive to prove the hypothesis that rosuvastatin may effectively reduce early recurrence of new ischemic lesions, probably due to insufficient sample size. We could not include a sufficient number of patients because of slow enrollment. Despite insufficient evidence that use of a statin is effective or safe in acute ischemic stroke, investigators were reluctant to administer placebo, since statin use on discharge became a performance measure for primary stroke center certification. ${ }^{19}$ However, a non-significant trend for less frequent appearance of new ischemic lesions on DWI or progression or clinical recurrence of stroke was observed in the rosuvastatin group. Thus, our findings support previous observational studies showing a benefit of statins in the acute stage of stroke., ${ }^{7,8}$

Previous randomized trials using statins in acute stroke included small numbers of patients and showed no clinical effect or even worse outcomes after statin treatment. ${ }^{20-22}$ Serial MRIs were used to determine outcomes in this study. Defining clinical recurrence of stroke is sometimes difficult during the acute stage because pre-existing symptoms often fluctuate or progress. Many ischemic lesions that are recognized on 
DWI during the acute stage of stroke are clinically silent, but they are direct surrogate markers of recurrence. Ischemic injury progresses during the acute stage of infarctions ${ }^{23}$ and may cause growth of infarct volume. By using MRI surrogate markers such as DWI and GRE, subclinical occurrence of ischemic and expansion of ischemic lesions as well as hemorrhagic outcomes might be assessed accurately.

In this study, new ischemic lesions in the placebo group developed less often than expectation which was assumed based on previous reports. ${ }^{13,14}$ This might be partly ascribed to the high frequency of use with dual or triple antiplatelet agents (about $75 \%$ ) in this study population. We hypothesized that the use of statins could reduce the risk of early recurrence of either symptomatic or asymptomatic ischemic lesions and expansion of ischemic lesions in acute stroke. This was because antithrombotic and anti-inflammatory effects of statins have been demonstrated in many experimental studies. Statins also play a beneficial role in stabilizing atherosclerotic plaques. ${ }^{6,24}$ In the subgroup analysis of our trial, the RR reduction of new DWI lesion occurrence in the rosuvastatin group was greater in patients who were more likely to have had an atherothrombotic infarction, such as a $20 \%$ reduction in patients with relevant artery stenosis $\geq 50 \%$ and $21 \%$ reduction in those with multiple lesions in the territory of relevant artery stenosis. In the PP population (excluding patients without relevant artery stenosis after assessment), the difference was much greater. Although it is possible that statins are more effective in the prevention of stroke with an atherothrombotic mechanism, this hypothesis was inconclusive in our study.

In this study, rosuvastatin $20 \mathrm{mg}$ was safe in that there were no differences in the development of adverse events. Notably, the occurrence of HI on GRE was remarkably reduced in the rosuvastatin group. In the Stroke Prevention by Aggressive Reduction in Cholesterol Levels trial, atorvastatin $80 \mathrm{mg}$ increased the risk of ICH (hazard ratio, 1.68; 95\% CI 1.092.59), ${ }^{2}$ and in the Heart Protection Study, simvastatin $40 \mathrm{mg}$ was associated with approximately 2 -fold increased risk of ICH in patients with prior stroke. ${ }^{10}$ However, statin use was not associated with $\mathrm{ICH}$ in a large cohort study in patients with recent ischemic stroke, ${ }^{25}$ and in a meta-analysis of primary and secondary prevention studies of statins..$^{26,27}$ Our findings suggest that statin use in the acute stage of stroke may protect against microvascular (capillary) damage and prevent HI. Matrix metalloproteinase- 9 is a key proteinase that mediates $\mathrm{HI}$ by disrupting microvascular integrity. ${ }^{28}$ Statins reduce expression of matrix metalloproteinase- 9 in endothelial cells, astrocytes, and human plasma. ${ }^{29,30}$ Thus, the protective effect of statins against occurrence of $\mathrm{HI}$ might be in part mediated by inhibiting matrix metalloproteinase- 9 .

This study has several limitations. First, this study did not include patients with cardioembolic sources and was conducted in one Asian country. Therefore, our results should be interpreted with caution. Second, the median NIHSS of the study population at screening was 3 , which suggests that enrolled patients had rather milder stroke. Although we assessed the outcome in the subgroup with significant stenosis of the relevant artery, this might affect the outcome such as the frequency of progression or recurrence of stroke. Furthermore, the primary outcome of this trial was not clinical stroke recurrence, but imaging-based recurrent ischemic lesions. As a result, the data of clinical stroke recurrence was captured based on the investigators' reports of adverse events. Finally, this study was stopped early due to slow enrollment, which resulted in underpowered results.

In conclusion, by using MRI surrogate markers such as DWI and GRE, subclinical occurrence of ischemic as well as hemorrhagic outcomes may be assessed very sensitively and accurately with a relatively smaller sample size. Further studies are required to elucidate the potential benefit of statins in acute stroke patients to conclusively support the routine use of statins.

\section{Acknowledgements}

Steering Committee: Ji Hoe Heo (Principle investigator, Chair), Dae Ryong Kang, Eung Yeop Kim, Jong S. Kim, Young Dae Kim, Byung Chul Lee, Kyung-Yul Lee, Hyo Suk Nam, Byung-Woo Yoon. Data Safety and Monitoring Board: Min Soo Park (Chair), Chung Mo Nam, Seung-Koo Lee, Kwang Ho Lee. Imaging Review and Adjudication: Dongbeom Song (Chair), Eung Yeop Kim, Jinna Kim, Eun-Hye Kim, Ki-Jeong Lee, Joonsang Yoo. Statistical Analysis: Youn Nam Kim. Enrolling Sites and Investigators: Severance Hospital: Ji Hoe Heo, Dong Hyun Lee, Jinkwon Kim, Tae-Jin Song; Samsung Changwon Hospital: Myoung-Jin Cha, Kyoung-Soo Lee; Kyung Hee University Hospital: Dae-Il Chang; Yeungnam University Medical Center: Jun Lee; Ewha Womans University Hospital: Yong-Jae Kim; Chunnam National University Hospital: Ki-Hyun Cho, Man-Seok Park, Joon-Tae Kim; Kangdong Hospital: Hye-Yeon Choi; Wonju Severance Hospital: Seo Hyun Km, Ji-Yong Lee; Sanggye Paik Hospital: Sang Won Han, Jae Hyeon Park; Changwon Fatima Hospital: Yo Han Jung, Jay-Cheol Kwon; Dongsan Medical Center: Sung-Il Sohn, Kyung-Hee Cho; Sacred Heart Hospital: Byung-Chul Lee, Mi Sun Oh; Samsung Medical Center: Oh Young Bang, Geyong-Moon Kim; Inha University Hospital: 
Joung-Ho Rha; Seoul National University Hospital: ByungWoo Yoon; Pusan Paik Hospital: Eung Gyu Kim; Hangang Sacred Heart Hospital: Yang Ki Minn; Gangnam Severance Hospital: Kyung-Yul Lee; Asan Medical Center: Jong S. Kim; Chosun University Hospital: Seong Hwan Ahn; National Medical Center: Jong Yun Lee; Konyang University Hospital: Kee Ook Lee; Korea University Hospital: Woo-Keun Seo; Yeouido St. Mary's Hospital: A-Hyun Cho; Pusan National University Hospital: Han-Jin Cho.

\section{References}

1. Baigent C, Keech A, Kearney PM, Blackwell L, Buck G, Pollicino C, et al.; Cholesterol Treatment Trialists' (CTT) Collaborators. Efficacy and safety of cholesterol-lowering treatment: prospective meta-analysis of data from 90,056 participants in 14 randomised trials of statins. Lancet 2005;366:1267-1278.

2. Amarenco P, Bogousslavsky J, Callahan A 3rd, Goldstein LB, Hennerici M, Rudolph AE, et al. High-dose atorvastatin after stroke or transient ischemic attack. N Engl J Med 2006;355: 549-559.

3. Tziomalos K, Giampatzis V, Bouziana SD, Spanou M, Kostaki $S$, Papadopoulou M, et al. Comparative effects of more versus less aggressive treatment with statins on the long-term outcome of patients with acute ischemic stroke. Atherosclerosis 2015;243:65-70.

4. Bourcier T, Libby P. HMG CoA reductase inhibitors reduce plasminogen activator inhibitor-1 expression by human vascular smooth muscle and endothelial cells. Arterioscler Thromb Vasc Biol 2000;20:556-562.

5. Schäfer A, Fraccarollo D, Eigenthaler M, Tas P, Firnschild A, Frantz $S$, et al. Rosuvastatin reduces platelet activation in heart failure: role of NO bioavailability. Arterioscler Thromb Vasc Biol 2005;25:1071-1077.

6. Sukhova GK, Williams JK, Libby P. Statins reduce inflammation in atheroma of nonhuman primates independent of effects on serum cholesterol. Arterioscler Thromb Vasc Biol 2002;22: 1452-1458.

7. Ní Chróinín D, Asplund K, Åsberg S, Callaly E, Cuadrado-Godia E, Díez-Tejedor E, et al. Statin therapy and outcome after ischemic stroke: systematic review and meta-analysis of observational studies and randomized trials. Stroke 2013;44:448-456.

8. Biffi A, Devan WJ, Anderson CD, Cortellini L, Furie KL, Rosand J, et al. Statin treatment and functional outcome after ischemic stroke: case-control and meta-analysis. Stroke 2011; 42:1314-1319.

9. Hong KS, Lee JS. Statins in acute ischemic stroke: a systematic review. J Stroke 2015;17:282-301.
10. Collins R, Armitage J, Parish S, Sleight P, Peto R; Heart Protection Study Collaborative Group. Effects of cholesterol-lowering with simvastatin on stroke and other major vascular events in 20536 people with cerebrovascular disease or other highrisk conditions. Lancet 2004;363:757-767.

11. Squizzato A, Romualdi E, Dentali F, Ageno W. Statins for acute ischemic stroke. Cochrane Database Syst Rev 2011;8:CD007551.

12. Jauch EC, Saver JL, Adams HP Jr, Bruno A, Connors JJ, Demaerschalk BM, et al. Guidelines for the early management of patients with acute ischemic stroke: a guideline for healthcare professionals from the American Heart Association/American Stroke Association. Stroke 2013;44:870-947.

13. Kang DW, Latour LL, Chalela JA, Dambrosia J, Warach S. Early ischemic lesion recurrence within a week after acute ischemic stroke. Ann Neurol 2003;54:66-74.

14. Kang DW, Kwon SU, Yoo SH, Kwon KY, Choi CG, Kim SJ, et al. Early recurrent ischemic lesions on diffusion-weighted imaging in symptomatic intracranial atherosclerosis. Arch Neurol 2007;64:50-54.

15. Chimowitz MI, Lynn MJ, Howlett-Smith H, Stern BJ, Hertzberg VS, Frankel MR, et al. Comparison of warfarin and aspirin for symptomatic intracranial arterial stenosis. $N$ Engl J Med 2005;352:1305-1316.

16. Ferguson GG, Eliasziw M, Barr HW, Clagett GP, Barnes RW, Wallace MC, et al. The North American Symptomatic Carotid Endarterectomy Trial: surgical results in 1415 patients. Stroke 1999;30:1751-1758.

17. Hacke W, Kaste M, Fieschi C, Toni D, Lesaffre E, von Kummer $\mathrm{R}$, et al. Intravenous thrombolysis with recombinant tissue plasminogen activator for acute hemispheric stroke. The European Cooperative Acute Stroke Study (ECASS). JAMA 1995; 274:1017-1025.

18. Nam HS, Lee KY, Han SW, Kim SH, Lee JY, Ahn SH, et al. Prediction of long-term outcome by percent improvement after the first day of thrombolytic treatment in stroke patients. $J$ Neurol Sci 2009;281:69-73.

19. Gorelick PB. Primary and comprehensive stroke centers: history, value and certification criteria. J Stroke 2013;15:78-89.

20. Kennedy J, Hill MD, Ryckborst KJ, Eliasziw M, Demchuk AM, Buchan AM; FASTER Investigators. Fast assessment of stroke and transient ischaemic attack to prevent early recurrence (FASTER): a randomised controlled pilot trial. Lancet Neurol 2007;6:961-969.

21. Beer C, Blacker D, Bynevelt M, Hankey GJ, Puddey IB. A randomized placebo controlled trial of early treatment of acute ischemic stroke with atorvastatin and irbesartan. Int J Stroke 2012;7:104-111.

22. Montaner J, Chacón P, Krupinski J, Rubio F, Millán M, Molina 
CA, et al. Simvastatin in the acute phase of ischemic stroke: a safety and efficacy pilot trial. Eur J Neurol 2008;15:82-90.

23. Iadecola C, Anrather J. The immunology of stroke: from mechanisms to translation. Nat Med 2011;17:796-808.

24. Aikawa M, Voglic SJ, Sugiyama S, Rabkin E, Taubman MB, Fallon JT, et al. Dietary lipid lowering reduces tissue factor expression in rabbit atheroma. Circulation 1999;100:1215-1222.

25. Hackam DG, Austin PC, Huang A, Juurlink DN, Mamdani MM, Paterson JM, et al. Statins and intracerebral hemorrhage: a retrospective cohort study. Arch Neurol 2012;69:39-45.

26. McKinney JS, Kostis WJ. Statin therapy and the risk of intracerebral hemorrhage: a meta-analysis of 31 randomized controlled trials. Stroke 2012;43:2149-2156.

27. Everett BM, Mora S, Glynn RJ, MacFadyen J, Ridker PM. Safety profile of subjects treated to very low low-density lipo- protein cholesterol levels $(<30 \mathrm{mg} / \mathrm{dl})$ with rosuvastatin 20 mg daily (from JUPITER). Am J Cardiol 2014;114:1682-1689.

28. Heo JH, Lucero J, Abumiya T, Koziol JA, Copeland BR, del Zoppo GJ. Matrix metalloproteinases increase very early during experimental focal cerebral ischemia. J Cereb Blood Flow Metab 1999; 19:624-633.

29. Sapienza P, Borrelli V, Sterpetti AV, Dinicola S, Tartaglia E, di Marzo L. Dose-dependent effect of rosuvastatin in the regulation of metalloproteinase expression. Ann Vasc Surg 2011;25: 823-829.

30. Wang S, Lee SR, Guo SZ, Kim WJ, Montaner J, Wang X, et al. Reduction of tissue plasminogen activator-induced matrix metalloproteinase- 9 by simvastatin in astrocytes. Stroke 2006;37: 1910-1912. 


\section{SUPPLEMENTARY DATA}

\section{Exclusion criteria}

The main exclusion criteria were hemorrhagic stroke or history of symptomatic hemorrhagic stroke; presence of high-risk potential cardiac sources of embolism or other determined etiology of stroke based on the Trial of Org 10,172 in Acute Stroke Treatment classification at the time of enrollment; known major hematologic, neoplastic, metabolic, gastrointestinal, or endocrine dysfunction; history of malignancy, except in subjects who had been disease-free $>5$ years or whose only malignancy has been basal or squamous cell skin carcinoma; life-threatening illness indicating that the subject is not expected to survive for at least 2 years; secondary causes of nephrotic syndrome and/or renal dysfunction (serum creatinine $>2.0 \mathrm{mg} / \mathrm{dL}$ ); uncontrolled hypertension defined as either a resting systolic blood pressure $>185 \mathrm{mmHg}$ or resting diastolic blood pressure $>110 \mathrm{mmHg}$ despite blood pressure lowering therapy; clinically significant heart disease likely to require coronary artery bypass surgery, cardiac transplantation, surgical repair, and/or valve replacement during the course of the study (within 14 days after enrollment); moderate or greater severity of congestive heart failure (New York Heart Association Class III or IV) or whose most recent determination of left ventricular ejection fraction was $<0.35$; triglyceride level $>500 \mathrm{mg} / \mathrm{dL}$; low-density lipoprotein (LDL) cholesterol level $>190 \mathrm{mg} / \mathrm{dL}$; creatine kinase $>3$ times the upper limit of normal range (ULN); aspartate aminotransferase, alanine aminotransferase, or bilirubin levels > 3 times the ULN; thyroid stimulating hormone > 1.5 times the ULN; modified Rankin scale score 4-6 before stroke; possible need for conventional angiography, intervention, or carotid artery surgery during the course of the study; known serious hypersensitivity reactions to HMG-CoA reductase inhibitors; and history of myopathy.

\section{Conduct of study}

The first patient was enrolled in August 2010, and the study was scheduled to complete enrollment in August 2012. However, in June 2013 the study was stopped early due to slow enrollment. All patient data were recorded on standardized data-collection forms by an investigator or coordinator who was unaware of study-group assignments. All data were subsequently entered into a web-based clinical data management system and managed by an independent data management service (ADM Korea Inc., Seoul, Korea).

Supplemental Table 1. Changes in lipid profiles before and after treatment

\begin{tabular}{lcrr}
\hline & Rosuvastatin $(n=155)$ & Placebo $(n=159)$ & $P$ value \\
\hline $\begin{array}{l}\text { Total cholesterol } \\
\text { Baseline }\end{array}$ & $196.8 \pm 37.6$ & $185.9 \pm 33.4$ & \\
Closing & $125.4 \pm 25.5$ & $183.2 \pm 34.6$ & $<0.008$ \\
Change & $-69.2 \pm 34.2$ & $-3.7 \pm 36.5$ & $<0.001$ \\
HDL cholesterol & & & \\
Baseline & $44.5 \pm 10.0$ & $44.3 \pm 12.3$ & 0.603 \\
Closing & $43.3 \pm 10.6$ & $41.9 \pm 12.4$ & 0.194 \\
Change & $-1.5 \pm 9.0$ & $-2.2 \pm 12.1$ & 0.477 \\
LDL cholesterol & & & \\
Baseline & $129.8 \pm 33.0$ & $119.9 \pm 29.7$ & 0.006 \\
Closing & $63 \pm 22.8$ & $115.1 \pm 31.3$ & $<0.001$ \\
Change & $-63.9 \pm 30.4$ & $-5.8 \pm 31.3$ & $<0.001$ \\
Triglyceride & & & \\
Baseline & $128.3 \pm 76.7$ & $132.6 \pm 65.9$ & 0.191 \\
Closing & $111.9 \pm 51.0$ & $165 \pm 100$ & $<0.001$ \\
Change & $-20.7 \pm 73.2$ & $29.2 \pm 86.1$ & $<0.001$ \\
\hline
\end{tabular}

HDL, high-density lipoprotein; LDL, low-density lipoprotein. 
Supplemental Table 2. Characteristic of excluded patients from mITT

\begin{tabular}{|c|c|c|c|c|c|c|}
\hline & \multicolumn{3}{|c|}{ Excluded from $\mathrm{mlTT}(\mathrm{n}=25)$} & \multicolumn{3}{|c|}{ Excluded from $\mathrm{mlTT}(\mathrm{n}=25)$} \\
\hline & $\begin{array}{l}\text { Excluded from } \mathrm{mlTT} \\
\qquad(\mathrm{n}=25)\end{array}$ & $\begin{array}{l}\text { Included in } \mathrm{mITT} \\
\qquad(\mathrm{n}=289)\end{array}$ & $P$ & $\begin{array}{l}\text { Rosuvastatin } 20 \mathrm{mg} \\
\qquad(\mathrm{n}=18)\end{array}$ & Placebo $(n=7)$ & $P$ \\
\hline \multicolumn{7}{|l|}{ Demographics } \\
\hline Sex (male) & $14(56.0)$ & $174(60.2)$ & 0.842 & $9(50.0)$ & $5(71.4)$ & 0.407 \\
\hline Age (year) & $67.4 \pm 11.8$ & $64.8 \pm 11.8$ & 0.299 & $65.7 \pm 11.1$ & $71.7 \pm 13.4$ & 0.260 \\
\hline Body mass index $\left(\mathrm{kg} / \mathrm{m}^{2}\right)$ & $23.2 \pm 3.3$ & $24.0 \pm 3.0$ & 0.184 & $23.4 \pm 3.1$ & $22.5 \pm 3.7$ & 0.551 \\
\hline Abdominal circumference $(\mathrm{cm})$ & $85.3 \pm 8.2$ & $86.8 \pm 9.4$ & 0.457 & $85.9 \pm 7.7$ & $83.8 \pm 10.1$ & 0.612 \\
\hline \multicolumn{7}{|l|}{ Past history } \\
\hline Hypertension & $19(76.0)$ & $187(64.7)$ & 0.357 & 13 (72.2) & $6(85.7)$ & 0.627 \\
\hline Diabetes mellitus & $12(48.0)$ & $89(30.7)$ & 0.123 & $9(50.0)$ & $3(42.8)$ & 1.000 \\
\hline Hypercholesterolemia & $6(24.0)$ & $44(15.2)$ & 0.256 & $5(27.7)$ & $1(14.2)$ & 0.627 \\
\hline Smoking & $17(68.0)$ & $158(54.6)$ & 0.281 & $12(66.6)$ & $5(71.4)$ & 1.000 \\
\hline Coronary artery occlusive disease & $2(8.0)$ & $3(1.0)$ & 0.053 & $1(5.5)$ & $1(14.2)$ & 0.49 \\
\hline Peripheral artery occlusive disease & $1(4.0)$ & $1(0.3)$ & 0.153 & $1(5.5)$ & $0(0.0)$ & 1.000 \\
\hline Previous stroke & $6(24.0)$ & $23(7.9)$ & 0.019 & $4(22.2)$ & $2(28.5)$ & 1.000 \\
\hline \multicolumn{7}{|l|}{ Concomitant medication } \\
\hline Antihypertensive & $14(56.0)$ & $126(43.5)$ & 0.324 & $9(50.0)$ & $5(71.4)$ & 0.407 \\
\hline Antiplatelet & & & 0.913 & & & 0.735 \\
\hline Aspirin & $5(20.0)$ & 73 (25.2) & & $3(16.6)$ & $2(28.5)$ & \\
\hline Clopidogrel & $2(8.0)$ & $17(5.8)$ & & 1 (5.5) & $1(14.2)$ & \\
\hline Aspirin and clopidogrel & $16(64.0)$ & $164(56.7)$ & & $12(66.6)$ & $4(57.1)$ & \\
\hline Aspirin and cilostazol & $1(4.0)$ & $13(4.4)$ & & $1(5.5)$ & $0(0.0)$ & \\
\hline Aspirin, clopidogrel, and cilostazol & $1(4.0)$ & $22(7.6)$ & & $1(5.5)$ & $0(0.0)$ & \\
\hline Anticoagulant & $0(0.0)$ & $1(0.3)$ & 1.000 & $0(0.0)$ & $0(0.0)$ & \\
\hline Lipid-lowering drug (other than statin) & $1(4.0)$ & $2(0.6)$ & 0.221 & $1(5.5)$ & $0(0.0)$ & 1.000 \\
\hline Diabetes mellitus drug & $11(44.0)$ & $58(20.0)$ & 0.010 & $8(44.4)$ & $3(42.8)$ & 1.000 \\
\hline NSAID & $3(12.0)$ & $18(6.2)$ & 0.228 & $2(11.1)$ & $1(14.2)$ & 1.000 \\
\hline Intravenous tPA & $1(4.0)$ & $8(2.7)$ & 0.531 & $0(0.0)$ & $1(14.2)$ & 0.28 \\
\hline Baseline DWI volume $\left(\mathrm{mm}^{3}\right)$ & 542.4 [203.5-4115.3] & 710 [259.1-2803.6] & 0.254 & 640.2 [223.7-4664.8] & $221.5[27.9-595.9]$ & 0.049 \\
\hline Baseline NIHSS & $4[2-5]$ & $3[1-6]$ & 0.206 & $3.5[2-5]$ & $4[2-8]$ & 0.544 \\
\hline \multicolumn{7}{|l|}{ Lab } \\
\hline White blood cells $\left(\times 10^{3} / \mu \mathrm{L}\right)$ & $7.898 \pm 2.436$ & $7.982 \pm 2.325$ & 0.896 & $8.248 \pm 2.74$ & $6.997 \pm 1.075$ & 0.296 \\
\hline Neutrophils $\left(\times 10^{3} / \mu \mathrm{L}\right)$ & $60.664 \pm 10.556$ & $61.921 \pm 17.159$ & 0.242 & $61.1 \pm 10.686$ & $59.543 \pm 10.96$ & 0.743 \\
\hline Hemoglobin (g/dL) & $13.480 \pm 1.720$ & $14.018 \pm 1.590$ & 0.098 & $13.461 \pm 1.716$ & $13.529 \pm 1.867$ & 0.936 \\
\hline Hematocrit (\%) & $39.416 \pm 4.776$ & $41.158 \pm 4.424$ & 0.054 & $39.656 \pm 5.024$ & $38.8 \pm 4.369$ & 0.737 \\
\hline Platelet count $\left(\times 10^{3} / \mu \mathrm{L}\right)$ & $238.96 \pm 74.077$ & $243.474 \pm 62.062$ & 0.630 & $253.167 \pm 81.4$ & $202.429 \pm 31.837$ & 0.086 \\
\hline BUN (mg/dL) & $16.108 \pm 5.354$ & $15.236 \pm 5.38$ & 0.387 & $15.444 \pm 4.93$ & $17.814 \pm 6.41$ & 0.388 \\
\hline Creatinine $(\mathrm{mg} / \mathrm{dL})$ & $0.894 \pm 0.296$ & $0.844 \pm 0.226$ & 0.399 & $0.807 \pm 0.198$ & $1.116 \pm 0.399$ & 0.020 \\
\hline Fasting glucose (mg/dL) & $137.656 \pm 60.42$ & $133.711 \pm 57.247$ & 0.728 & $131.411 \pm 47.397$ & $153.714 \pm 88.428$ & 0.556 \\
\hline Albumin (g/dL) & $4.012 \pm 0.461$ & $4.127 \pm 0.336$ & 0.200 & $3.978 \pm 0.479$ & $4.1 \pm 0.432$ & 0.544 \\
\hline Uric acid (mg/dL) & $5.146 \pm 1.616$ & $5.052 \pm 1.429$ & 0.812 & $4.831 \pm 1.609$ & $5.957 \pm 1.427$ & 0.088 \\
\hline $\mathrm{hsCRP}(\mathrm{mg} / \mathrm{dL})$ & $2.843 \pm 6.94$ & $2.266 \pm 6.955$ & 0.783 & $2.79 \pm 7.423$ & $2.98 \pm 6.042$ & 0.956 \\
\hline
\end{tabular}

Values are number (\%), mean \pm standard deviation, or median [interquartile range].

mITT, modified intention-to-treat; NSAID, indicates nonsteroidal anti-inflammatory drug; t-PA, tissue plasminogen activator; DWI, diffusion-weighted imaging; NIHSS, National Institute of Health Stroke Scale; hsCRP, high sensitivity C-reactive protein; BUN, blood urea nitrogen. 